ARTICLE

Received 15 Jun 2016 | Accepted 3 Nov 2016 | Published 13 Dec 2016

DOI: $10.1038 /$ ncomms13837

OPEN

\title{
ATR inhibitors as a synthetic lethal therapy for tumours deficient in ARID1A
}

Chris T. Williamson ${ }^{1,2}$, Rowan Miller ${ }^{1,2}$, Helen N. Pemberton ${ }^{1,2}$, Samuel E. Jones ${ }^{1,2}$, James Campbell ${ }^{1,2}$, Asha Konde1,2, Nicholas Badham 1,2, Rumana Rafiq ${ }^{1,2}$, Rachel Brough 1,2, Aditi Gulati1,2, Colm J. Ryan ${ }^{3}$, Jeff Francis ${ }^{1,2}$, Peter B. Vermulen ${ }^{2,4}$, Andrew R. Reynolds ${ }^{2}$, Philip M. Reaper ${ }^{5}$, John R. Pollard ${ }^{5}$, Alan Ashworth ${ }^{1,2, \dagger} \&$ Christopher J. Lord ${ }^{1,2}$

Identifying genetic biomarkers of synthetic lethal drug sensitivity effects provides one approach to the development of targeted cancer therapies. Mutations in ARID1A represent one of the most common molecular alterations in human cancer, but therapeutic approaches that target these defects are not yet clinically available. We demonstrate that defects in ARID1A sensitize tumour cells to clinical inhibitors of the DNA damage checkpoint kinase, ATR, both in vitro and in vivo. Mechanistically, ARID1A deficiency results in topoisomerase 2A and cell cycle defects, which cause an increased reliance on ATR checkpoint activity. In ARID1A mutant tumour cells, inhibition of ATR triggers premature mitotic entry, genomic instability and apoptosis. The data presented here provide the pre-clinical and mechanistic rationale for assessing ARID1A defects as a biomarker of single-agent ATR inhibitor response and represents a novel synthetic lethal approach to targeting tumour cells.

\footnotetext{
${ }^{1}$ The CRUK Gene Function Laboratory, The Institute of Cancer Research, London SW3 6JB, UK. ${ }^{2}$ The Breast Cancer Now Toby Robins Breast Cancer Research Centre, The Institute of Cancer Research, London SW3 6JB, UK. ${ }^{3}$ Systems Biology Ireland, University College Dublin, Dublin 4, Ireland. ${ }^{4}$ GZA Hospitals Sint-Augustinus, Wilrijk, Belgium and Center for Oncological Research, University of Antwerp, Oosterveldlaan 24, Wilrijk Antwerp 2610, Belgium.

${ }^{5}$ Vertex Pharmaceuticals (Europe) Limited, Milton Park, Abingdon, Oxfordshire OX14 4RY, UK. † Present address: UCSF Helen Diller Family Comprehensive Cancer Centre, San Francisco, California 94158, USA. Correspondence and requests for materials should be addressed to A.A. (email: Alan.Ashworth@ucsf.edu) or to C.J.L. (email: Chris.Lord@icr.ac.uk).
} 
TR (Ataxia-Telangiectasia Mutated (ATM) and Rad3related protein kinase), is a critical component of the cellular DNA damage response (DDR) ${ }^{1}$. ATR is activated by regions of single-stranded DNA, some of which occur as a result of replication stress ${ }^{2-4}$. Oncogene activation can induce replication stress and a reliance upon an ATR checkpoint function; this provides one rationale for the use of small molecule ATR inhibitors (ATRi) as cancer therapeutics ${ }^{5}$. Potent and specific ATRi have been discovered including EPT-46464 (ref. 6), AZ20 (AstraZeneca) ${ }^{7}, \mathrm{VE}-821$ and VX-970 (VE-822) (Vertex), some of which are currently in Phase I clinical trials ${ }^{5}$. In pre-clinical studies, VE-821 enhances the cytotoxic effects of a number of DNA damaging agents in tumour cells that have defects in the ATM/p53 pathway ${ }^{8-11}$, suggesting that ATRi might have clinical utility as chemo-sensitizing agents. However, in what context ATRi might be used as single agents is less clear. Previous studies have demonstrated that alterations in canonical DDR/cell cycle checkpoint genes (ERCC1 (ref. 12), XRCC1 (ref. 13), CDC25 $A^{14}$ and $A T M^{15,16}$ ) have the potential to act as predictive biomarkers of single-agent ATRi sensitivity. However, it is not yet clear whether processes beyond canonical DDR, and in particular loss of tumour suppressor genes, might also predict for ATRi sensitivity. Therefore, as ATRi enter Phase 1 clinical trials, it is clear that there is a pressing need to identify clinically useful biomarkers of sensitivity ${ }^{5}$.

The SWI/SNF chromatin-remodelling complex is composed of multiple components, including proteins such as ARID1A, ARID1B, SMARCA4 and SMARCB1 that have tumour suppressor roles ${ }^{17}$. These complexes utilize ATP to modify chromatin architecture by sliding or ejecting nucleosomes from $\mathrm{DNA}^{18}$. This activity appears to modulate a number of DNA processes including replication, transcription and DNA repair ${ }^{19,20}$. Two primary versions of SWI/SNF have been isolated from cells, BAF (SWI/SNF-A) and PBAF (SWI/SNF-B) ${ }^{21}$, distinguished in part by the DNA-binding component of the complex. BAF complexes interact with DNA via either ARID1A or ARID1B components, while the PBAF complex binds DNA through ARID2 (ref. 22). When taken as a group, SWI/SNF components are estimated to be mutated in nearly $20 \%$ of all human tumours, making loss of this complex one of the most common alterations in cancer ${ }^{17}$.

In this study, we aimed to discover clinically actionable determinants of single-agent ATRi sensitivity. Using large-scale genetic screens we identified the BAF component ARID1A as a synthetic lethal partner of ATR inhibition. We validated the synthetic lethal interaction between ATR and ARID1A using both in vitro and in vivo models. Mechanistically, we found that ATR inhibition exploits a pre-existing DNA decatenation defect in ARID1A mutant tumour cells and causes premature mitotic progression. This leads to large-scale genomic instability and cell death. On the basis of this data, we propose that ARID1A should be assessed as a biomarker of ATRi sensitivity in clinical trials.

\section{Results}

RNAi screens identify ARID1A as ATRi synthetic lethal partner. To uncover clinically actionable genetic determinants of singleagent ATRi response, we performed a series of high-throughput RNAi chemosensitization screens where cells were transfected with a library of SMARTPool short interfering (si)RNAs and then exposed to the highly potent and selective ATR catalytic inhibitor VE-821 (Fig. 1a; $K_{\mathrm{i}}=13 \mathrm{nM}$ (ref. 23)). For screening we selected the p53 mutant, triple negative (ER $\alpha$ negative, $\mathrm{PR}$ negative and ERBB2 negative) breast tumour cell line HCC1143, based on previous work suggesting that ATRi might have utility in TP53 mutant cancers ${ }^{6,9,24,25}$. To model the effect of ATRi on normal cells, we also screened the non-tumour, mammary epithelial cell model, MCF12A. We confirmed that both cell lines retained a functional ATR activation pathway by assessing cisplatininduced ATR p.T1989 autophosphorylation ${ }^{26,27}$ (Supplementary Fig. 1A,B). To identify clinically actionable effects, the RNAi library we used encompassed 1,280 siRNA SMARTPools (four siRNAs per gene in each pool) targeting either recurrently mutated genes in cancer ${ }^{28}$, kinases, due to their inherent tractability as drug targets, and DDR genes ${ }^{29}$, given the potential for ATRi to enhance defects in these processes ${ }^{6,9}$ (Supplementary Data 1). HCC1143 and MCF12A cells were transfected in a 384well plate format using the siRNA library. Cells were then exposed to a sub-lethal concentration of VE-821 (1 $\mu \mathrm{M}$, Supplementary Fig. 1C) or vehicle (DMSO) for a subsequent 4 days, at which point cell viability was estimated using CellTitre-Glo Reagent (Promega; Fig. 1a).

We used data from replica $(n=3)$ screens to calculate drug effect (DE) $Z$-scores (see Methods) describing the effect of each siRNA SMARTPool on VE-821 sensitization (Fig. 1b) ${ }^{30}$. This approach identified 150 significant $(\mathrm{DE} Z<-2)$ VE-821 sensitivity-causing effects in HCC1143 cells and 179 in MCF12A cells (Fig. 1b,c and Supplementary Data 2). Consistent with previous observations ${ }^{9,12,15,16}$, we observed significant VE-821 sensitization associated with siRNA SMARTPools designed to target either $A T R$, the ATR activation factors RAD17, RAD1 and $R A D 9 A, E R C C 4$ or ATM (Supplementary Fig. 1D,E), giving us confidence in the results from the screens.

To identify ATRi synthetic lethal effects operating in diverse genetic backgrounds, we compared the HCC1143 and MCF12A data and identified 30 siRNA SMARTPools that caused VE-821 sensitivity in both cell lines (Supplementary Data 2). This analysis identified several novel ATR synthetic lethal partner genes involved in DNA damage/repair including those targeting components of the HR/Fanconi Anaemia pathway (FANCE, SLX4, PALB2), DNA mismatch repair (MSH4, PMS2) and trans-lesion synthesis (RAD18) pathways (Fig. 1c and Supplementary Data 2). Amongst the common hits we also identified seven siRNA targeting tumour suppressor genes (ATM, FANCE, GCP3, IDH1, PALB2, PMS2 and ARID1A; Fig. 1c,d). The observation that silencing ARID1A sensitized cells to ATRi was particularly interesting as $A R I D 1 A$ is recurrently mutated in a variety of tumour types (45\% ovarian clear cell carcinoma (OCCC), 14-19\% gastric, bladder and hepatocellular tumours and $2-3 \%$ breast tumours ${ }^{17}$ ).

We validated the ARID1A/ATRi synthetic lethal interaction using a number of orthogonal approaches. In HCC1143 cells, an siRNA SMARTPool targeting ARID1A-reduced ARID1A protein levels and significantly enhanced sensitivity to VE-821 $(P<0.0001$, analysis of variance (ANOVA), Fig. $1 \mathrm{e}, \mathrm{f})$ as did the four individual ARID1A siRNAs $(P<0.001$, Student's $t$-test, Fig. 1g,h), suggesting that this was unlikely to be an off-target siRNA effect. Both pooled and individual ARID1A siRNAs also caused sensitivity to an ATRi currently being assessed in Phase 1 clinical trials trials, VX-970 (Fig. 2a), suggesting that the synthetic lethality was not specific to VE-821 (Supplementary Fig. 2A,B). To assess whether constitutive loss of ARID1A function, as would occur in tumours, also resulted in ATRi sensitivity, we used human colorectal HCT116 isogenic cells containing either wildtype ARID1A (ARID1A $\left.{ }^{+/+}\right)$or homozygous loss-of-function ARID1A mutations (p.Q456*/p.Q456*; referred to as ARID1A ${ }^{-/-}$; Fig. 2b). In clonogenic survival assays exposure to three distinct ATRi (VE-821, VX-970 and AZ20) all significantly impaired the survival of $A R I D 1 A^{-/}$cells compared with $A R I D 1 A^{+/+}(P<0.0001$, ANOVA, Fig. 2c-f $)$. The ARID1A ${ }^{-/-}$selective toxicity of ATRi was also observed in short-term (5-day exposure) viability assays using all three inhibitors $(P<0.001$, ANOVA, Fig. $2 \mathrm{~g}-\mathrm{i})$. In contrast to our 


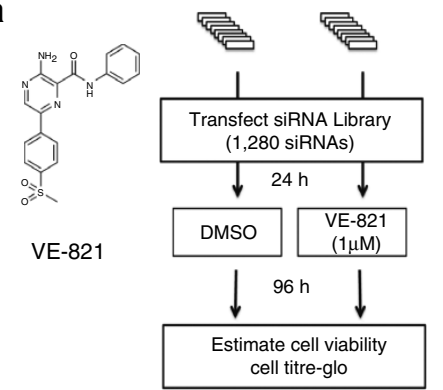

c

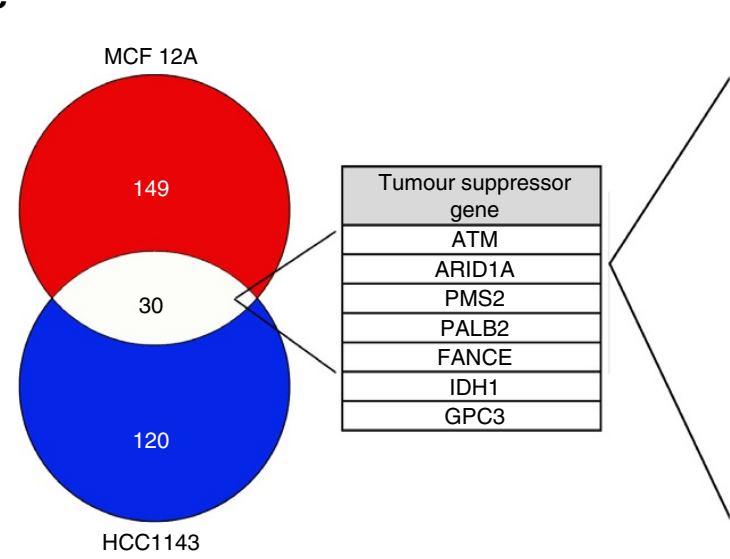

MCF 12A

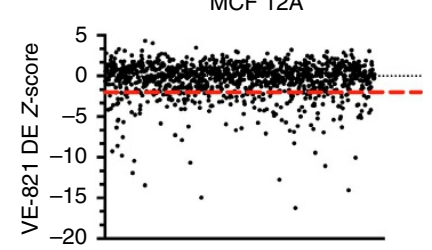

b

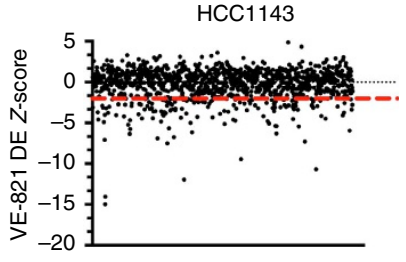

d
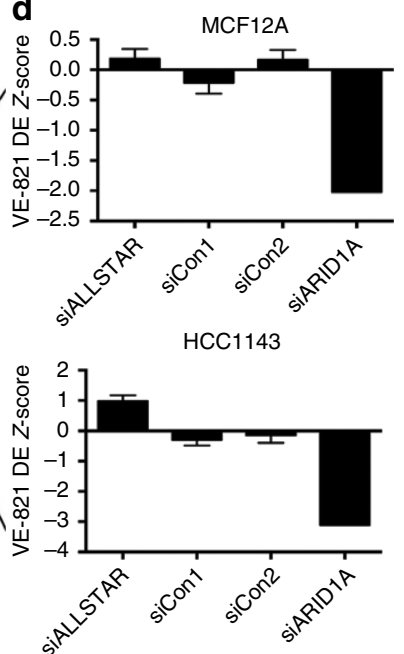

e
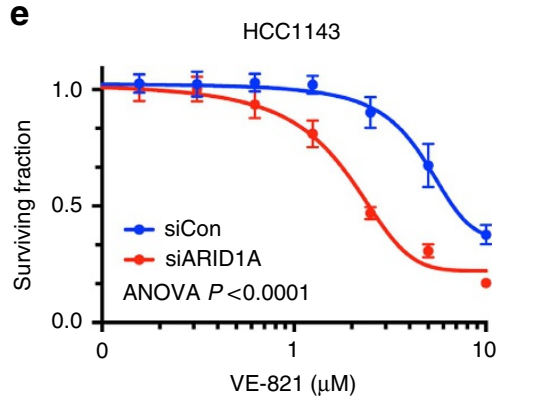

f $\mathrm{HCC} 1143$

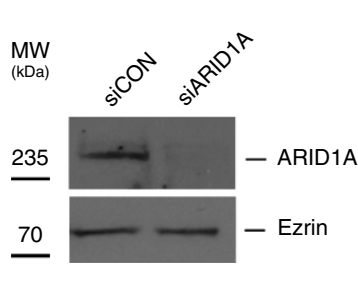

g

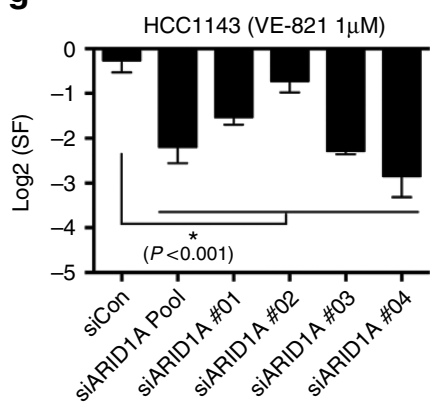

h

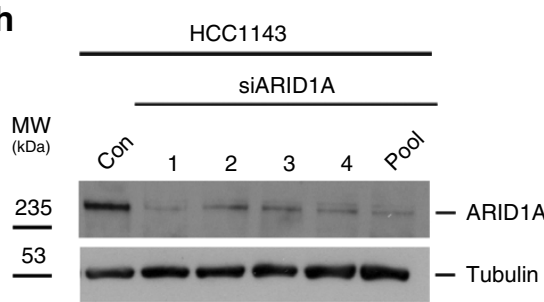

Figure 1 | RNAi screen reveals genetic determinants of ATRi sensitivity. (a) Structure of VE-821 and schematic representation describing workflow for parallel VE-821 chemosensitization screens in MCF12A and HCC1143 cells. (b) Scatter plots of VE-821 Drug Effect (DE) Z-scores from all 1280 siRNAs SMARTpools used in the chemosensitization screens. The DE Z-score threshold of -2 (dotted red line) was used for defining candidate/unvalidated synthetic lethal interactions. (c) Venn diagram of DE $<-2$ VE-821 sensitization hits in MCF12A and HCC1143 cells. Numbers shown indicate number of sensitization genes. Amongst the 30 genes with DE Z-scores $<-2$ in both cell lines, seven well-established tumour suppressor genes were identified. (d) Bar charts illustrating DE Z-scores for control, non-targeting, siRNAs (siALLSTAR, siCon1, siCon2) and ARID1A SMARTPool siRNAs in the chemosensitization screens. Values shown are medians from triplicate screens. Error bars represent s.d. (e) Three-hundred eighty-four-well plate cell survival data from HCC1143 cells transfected with siRNA targeting ARIDIA (red) or siCon (blue). Twenty four hours after transfection, cells were exposed to VE-821 for 5 continuous days. Error bars represent s.d. $(n=16)$ and results are representative of three biological replicates. Survival curve siARID1A versus siCon $P$ value $<0.0001$, ANOVA. (f) Western blot illustrating ARID1A protein silencing from experiment (e). (g) Bar chart illustrating the Log2 surviving fractions (Log2(SF)) of HCC1143 cells transfected with the indicated individual siRNAs and exposed to VE-821 ( $1 \mu \mathrm{M})$ for 5 days. Error bars represent s.d. and $P$ values of $<0.001$, Student's t-test, for each siRNA compared with siCon. (h) Western blot illustrating ARID1A protein silencing from experiment (g). 
a

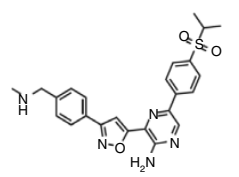

VX-970 b

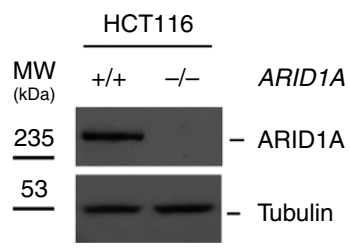

c

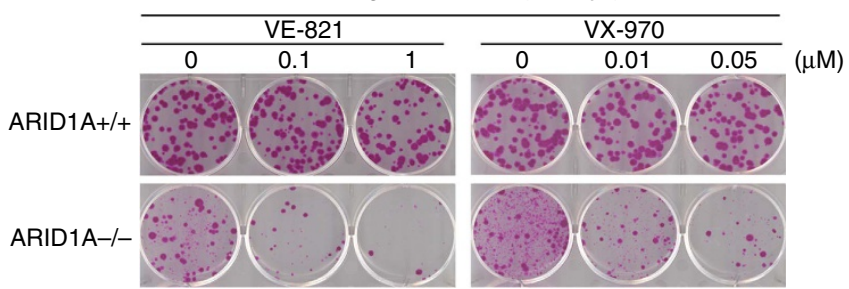

d
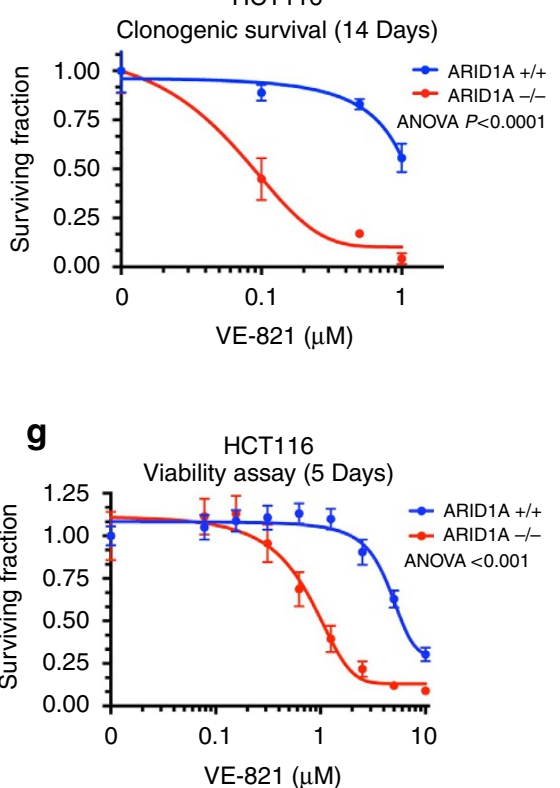

j

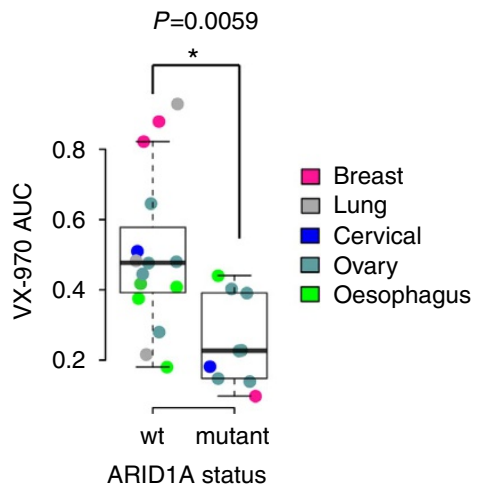

e

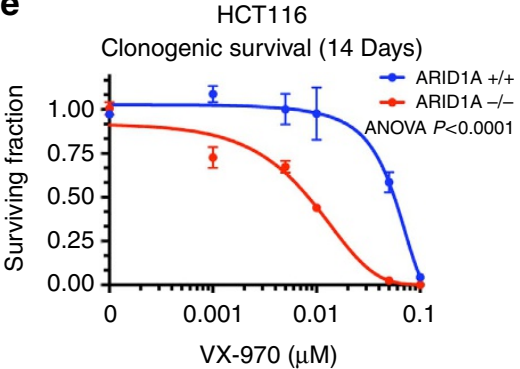

h

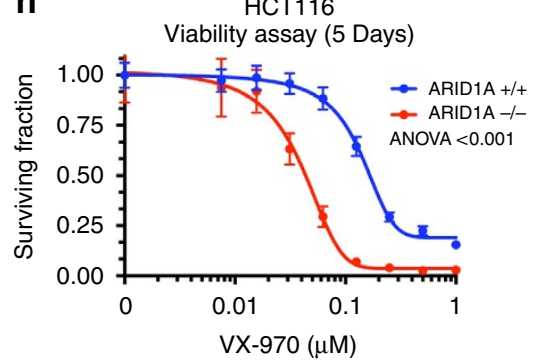

k

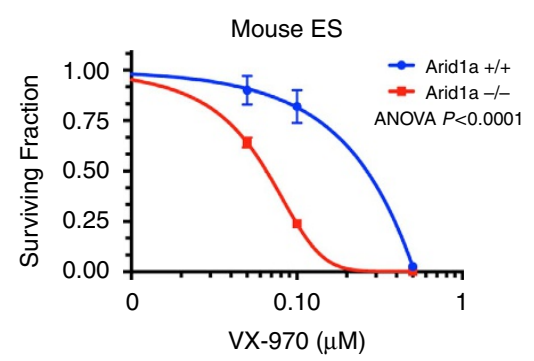

f

Clonogenic survival (14 Days)

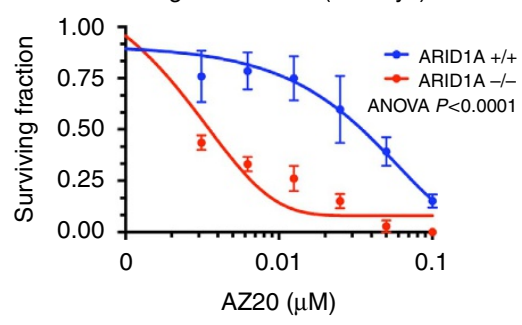

i

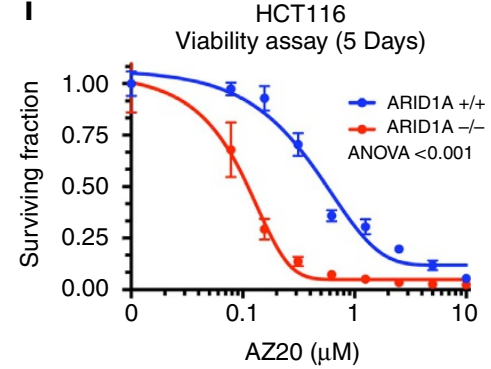

I

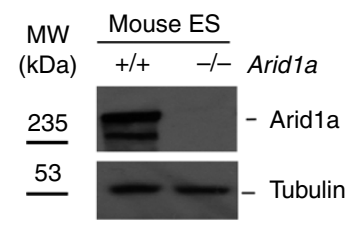

Figure 2 | In vitro ARID1A/ATR synthetic lethality. (a) Chemical structure of VX-970. (b) Western blot of ARID1A in human ARID1A isogenic HCT116 cells. (c) Image of colonies in six-well-plate clonogenic assay. HCT116 ARID1A isogenic $(+/+$ and $-/-)$ cell lines were exposed to increasing concentrations of VE-821 $(0,0.1,1 \mu \mathrm{M})$ or $\mathrm{VX}-970(0,0.01,0.05 \mu \mathrm{M})$ for 14 days. (d-f) Dose-response clonogenic survival curves of HCT116 ARID1A isogenic $(+/+$ and $-/-$ ) cell lines exposed to increasing concentrations of VE-821 (d), VX-970 (e) and AZ-20 (f) for 14 days. Error bars represent s.d. $(n=3)$, ANOVA $P$ value of $<0.0001$, results are representative of triplicate biological experiments. (g-i) Dose-response survival curves of HCT116 ARID1A isogenic $(+/+$ and $-/-$ ) cell lines exposed to increasing concentrations of VE-821 (g), VX-970 (H) or AZ-20 (I) for 5 days. Cell viability was estimated by CellTitre-Glo reagent. Error bars represent s.d. $(n=16)$, ANOVA $P$ value of $<0.001$, results are representative of triplicate biological experiments. (j) Area under curve (AUC) box whisker comparison plot for human tumour cell lines exposed to VX-970 for 5 days. ARID1A wild-type tumour cell lines $(n=15)$ were compared with ARID1A mutant cell lines $(n=9) . P=0.00594$ median permutation test. (k). Dose-response clonogenic survival curve of mouse Arid1a isogenic ES cell lines. Experiment was performed as per $(\mathbf{c})$. Error bars represent s.d. $(n=3)$, ANOVA $P$ value of $<0.0001$, results are representative of triplicate biological experiments. (I). Western blot of Aridla protein expression in mouse ES Aridla isogenic cells.

observations using ATRi, HCT116 ARID1A isogenic cells had a similar level of sensitivity to other common chemotherapeutic agents such as methotrexate and taxol, suggesting that
ARID $1 A^{-/-}$cells are not globally sensitive to exogenous agents (Supplementary Fig. 2C,D). In addition, we noted that heterozygous HCT116 ARID1A ${ }^{+/-}$cells expressed an intermediate 
level of ARID1A protein compared with homozygous wild-type and homozygous mutant cells and exhibited an intermediate level of ATRi sensitivity (Supplementary Fig. 2E,F). This suggested that an ARID1A gene dosage effect might possibly influence ATRi sensitivity.

The ATRi sensitivity in HCT116 ARID1A ${ }^{-/-}$cells not only validated the synthetic lethal interaction between ARID1A and ATR inhibition but also established that the effect was likely not restricted to cells from a breast lineage such as HCC1143 or MCF12A used in the high-throughput screens. To further investigate the generality of this effect we determined ATRi sensitivity in a genetically and histologically diverse panel of tumour cell lines. In this analysis, we found that the presence of loss-of-function ARID1A mutations in tumour cell lines was associated with sensitivity to VX-970 $(P=0.0059$, Student's $t$-test, Fig. $2 \mathrm{j}$ and Supplementary Data 3 ), although we were statistically underpowered to confirm whether or not the synthetic lethality was any more or less profound in particular tumour types in this analysis. To address whether the ATR/ARID1A synthetic lethal interaction was limited to human cell line models, we also studied isogenic Aridla $a^{+1+}$ and Aridla $a^{-1-}$ mouse embryonic stem (ES) cells ${ }^{31}$. In clonogenic assays both VX-970 and VE-821 selectively targeted Arid1a $a^{-1-}$ ES cells $(P<0.0001$, ANOVA, Fig. 2k,l and Supplementary Fig. 2E). We next compared how the observed ATR/ARID1A synthetic lethality compared with other ATRi related synthetic lethalities and other reported ARID1Aselective agents. The magnitude of ATRi sensitivity associated with ARID1A mutant cells was comparable, if not greater than that seen with other candidate biomarkers of ATRi sensitivity such as ATM (Supplementary Fig. 2F). Furthermore, we found that the ARID1A - - selectivity of VX-970 was also more profound than other proposed ARID1A-targeted agents such as PARP inhibitors (PARPi) or cisplatin ${ }^{32,33}$ (Supplementary Fig. 3).

We originally identified the ATR/ARID1A synthetic lethal effect in p53 mutant HCC1143 and p53 wild-type MCF12A cells and confirmed this synthetic lethality in p53 wild-type HCT116 and mouse ES cells (Figs 1 and 2). This suggested that the synthetic lethal effect was somewhat independent of p53 status. Silencing of ARID1A using siRNA significantly sensitized HCT116 TP53 ${ }^{-/}$- cells to VX-970 $(P<0.001$, ANOVA, Supplementary Fig. 4A), suggesting that the absence of p53 function did not negate the ATR/ARID1A synthetic lethality. In addition, silencing of TP53 using siRNA in p53 wild-type HCT116 ARID1A $A^{-1}$ - cells also did not alter the sensitivity to VX-970 (Supplementary Fig. 4B). Collectively this data suggested that p53 might not be a key modulator of the ATR/ARID1A synthetic lethality.

To address the possibility that the ARID1A/ATRi synthetic lethality might be specific to catalytic inhibition of ATR, we exploited data describing the sensitivity of 86 human tumour cell lines to SMARTPool siRNAs targeting 720 kinase-coding genes $^{34,35}$. We found that the presence of loss-of-function ARID1A mutations in tumour cell lines was associated with greater sensitivity to ATR siRNA ( $P=0.008$, Median Permutation test, Supplementary Fig. 4C). To confirm this, we silenced ATR using siRNA in HCT116 ARID1A ${ }^{-/-}$and ARID1A $A^{+/+}$cells, and found that ATR siRNA selectively targeted ARID1A ${ }^{-/-}$cells $(P<0.05$, Student's $t$-test, Supplementary Fig. 4D,E). Collectively, this data suggested that inhibition of ATR function, either by small molecule inhibition or by gene silencing was synthetically lethal with ARIDIA deficiency.

In vivo ATR/ARID1A synthetic lethality. We next assessed whether the clinical ATRi, VX-970, could inhibit ARID1Adeficient tumours in vivo. To do this, we generated cohorts of mice with established xenograft tumours derived from either HCT116 ARID1A ${ }^{+/+}$or ARID $1 A^{-/-}$cells. Once tumours had established, mice were treated with either VX-970 or drug vehicle for 40 days (Fig. 3a). We found that, compared with vehicle treatment, VX-970 had no effect on ARID1A ${ }^{+/+}$tumours $(P=0.45$, ANOVA, Fig. 3b) but significantly inhibited the growth of ARID1A $A^{-/-}$tumours $(P=0.024$, ANOVA, Fig. 3b). VX-970 treatment also had a greater impact on $A R I D 1 A^{-/-}$tumours compared with $A R I D 1 A^{+/+}$tumours $(P=0.006$, ANOVA, Fig. 3b). In a subsequent experiment, we assessed whether VX-970 could impair the establishment of tumours by treating mice with VX-970 immediately after xenografting HCT116 cells, as opposed to waiting for tumours to establish before initiating treatment (Fig. 3c). We found that VX-970 prevented the establishment of HCT116 ARID1A ${ }^{-/-}$xenografts (frequency of ARID $1 A^{-1-}$ tumour formation $=27 \%$ for VX-970-treated mice versus $73 \%$ in vehicle-treated mice, $P=0.027$, Fisher's exact test), but had no impact on the establishment of ARID1A $+/+$ xenografts (frequency of $A R I D 1 A^{-/-}$tumour formation $=80 \%$ for VX-970-treated mice versus $87 \%$ in vehicle-treated mice, $P=1 \mathrm{~ns}$ by Fisher's exact test, Fig. 3c,d). In this experiment, early initiation of VX-970 treatment also dramatically slowed the growth of $A R I D 1 A^{-/-}$tumours $(P=0.015$, ANOVA), but did not impair ARID $1 A^{+/+}$xenografts $(P=0.63$, ANOVA, Fig. $3 \mathrm{e}, \mathrm{f})$. We also determined the in vivo efficacy of VX-970 in mice with established tumours derived from a tumour cell line with naturally occurring ARID1A mutations (TOV21G, ARID1A p.548fs/p.756fs). In in vitro studies, we found that the TOV21G human OCCC cell line was more sensitive to both VE-821 and VX-970 than the ARID1A wild-type OCCC cell line RMG1 (Fig. 3g, $P<0.0001$, ANOVA and Supplementary Fig. 4F) and also exhibited a DNA damage ( $\gamma \mathrm{H} 2 \mathrm{AX}$; Supplementary Fig. $4 \mathrm{G})$ and apoptotic response to ATRi exposure (Fig. 3h,i). In vivo, VX-970 treatment significantly inhibited the growth of established TOV21G tumours compared with vehicle administration $(P=0.014$, ANOVA, Fig. $3 \mathrm{j}-1)$. Although the scale of anti-tumour effect in TOV21G xenografts was not as profound as in HCT116 ARID1A ${ }^{-/-}$xenografts, the effect of ATRi in this setting did suggest that the ARID1A/ATR synthetic lethality could be exploited in vivo and warrants further investigation.

ARID1A loss results in TOP2A and cell cycle defects. In the course of establishing what differences between HCT116 ARID $1 A^{+/+}$and ARID $1 A^{-/-}$cells could explain the selective toxicity of ATR inhibition, we found that under logarithmic growth conditions, and in the absence of ATRi, the proportion of $\mathrm{G}_{2} / \mathrm{M}$ cells was higher in $A R I D 1 A^{-/-}$cells compared with ARID $1 A^{+/+}$cells $(P=0.033$, Student's test, Fig. $4 \mathrm{a})$. To investigate this further, we synchronized cells at the $G_{1}$ /early $S$ phase boundary using a double thymidine block and then followed cell cycle progression once the thymidine block was removed (Fig. 4b). We found that, compared with $A R I D 1 A^{+/+}$cells, ARID1A $A^{-/-}$cells had a delayed progression through $\mathrm{S}$ phase and a slower progression from $\mathrm{G}_{2}$ into mitosis $(8-10 \mathrm{~h}$ after block-Fig. 4b). In asynchronous cell cultures, we found that ARID1A ${ }^{-/-}$cells exhibited lower levels of phosphorylated Histone H3 (Ser-10), a marker of mitotic entry, compared with ARID $1 A^{+/+}$cells $(P=0.035$, Student's $t$-test, Fig. $4 c)$. Silencing of $A R I D 1 A$ by siRNA SMARTpool in $A R I D 1 A^{+/+}$cells also caused a significant decrease in Histone $\mathrm{H} 3$ phosphorylation $(P=0.00015$, Student's $t$-test, Fig. $4 c)$. We did however note that the extent of phospho-H3 reduction caused by ARID1A inhibition was more extensive in ARID1A siRNA SMARTpool transfected cells than in ARID1A ${ }^{-/-}$cells (Fig. 4c). It is possible that this could be due to a modest difference in the pheno- 
a

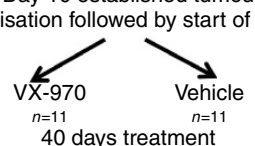

C

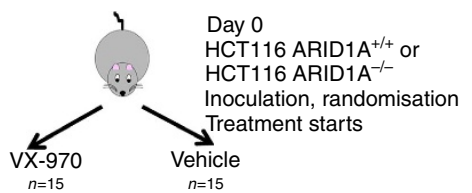

40 days treatment

e

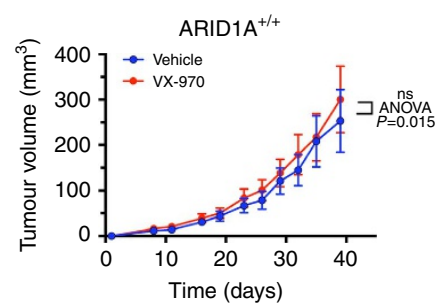

b
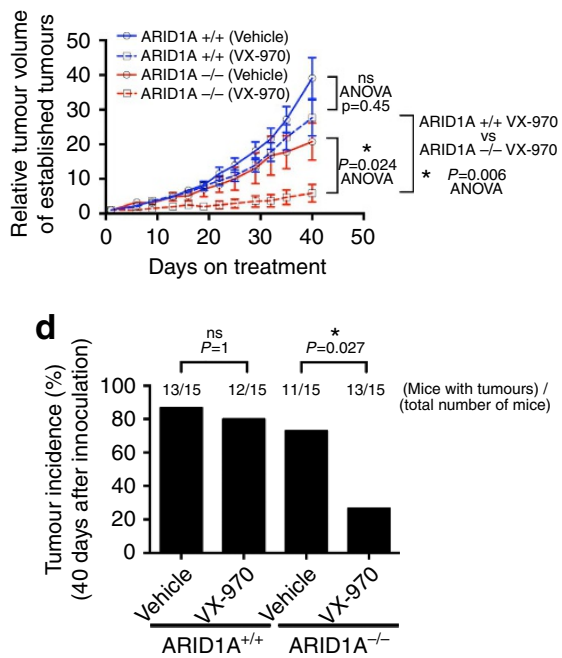

f

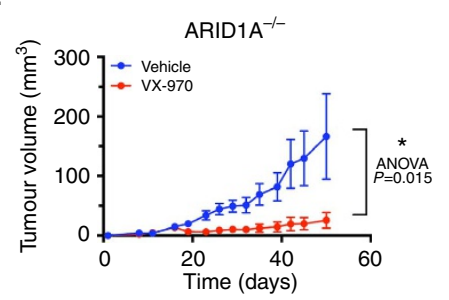

g

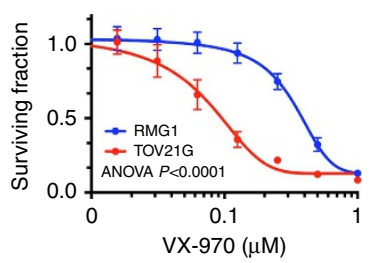

j

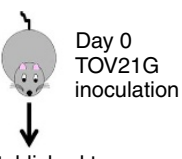

Day 8 Established tumours

Randomization followed by starts of treatment

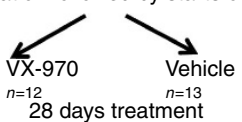

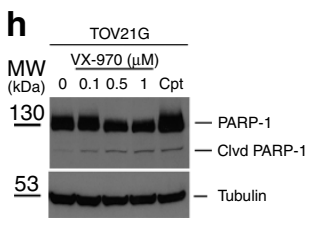

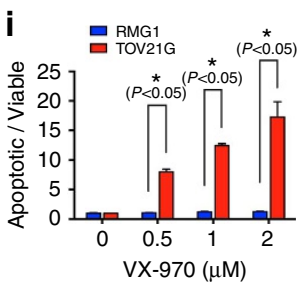

k

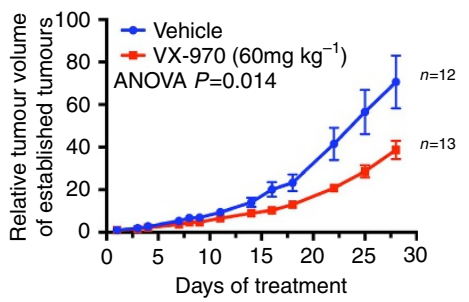

I

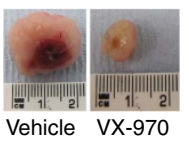

Figure 3 | In vivo ARID1A/ATR synthetic lethality. (a). Schematic representation of VX-970 therapy experiment in mice bearing established HCT116 $A R I D 1 A^{+/+}$and ARIDIA ${ }^{-/-}$xenografts. Mice were then randomized to treatment cohorts of either $\mathrm{VX}-970\left(60 \mathrm{mg} \mathrm{kg}^{-1}, 4 \times\right.$ weekly by oral gavage) or vehicle treatments. $N=11$ mice in each cohort. Mice were treated for a subsequent 40 days. Tumour volume was monitored thrice weekly. (b) Relative tumour volume plot from experiment (A) showing efficacy of VX-970 and selectivity for $A R I D 1 A^{-/-}$xenografts ${ }^{*} P=0.024$, ANOVA, and a greater efficacy of VX-970 on ARIDIA ${ }^{-/-}$xenografts compared with ARID1A $+/+$xenografts ${ }^{\star} P=0.006$, ANOVA. (c) Schematic representation of tumour incidence experiment in mice with non-established HCT116 ARID1A ${ }^{+/+}$and $A R I D 1 A^{-/-}$xenografts. $N=15$ mice per cohort. Tumour volume was monitored thrice weekly and overall tumour incidence was assessed 40 days later. (d) Bar chart of tumour incidence 40 days after implantation of HCT116 ARIDIA ${ }^{+/+}$or $A R I D 1 A^{-/-}$cells. Numbers above bars indicate the proportion of animals in which a detectable xenograft formed. ${ }^{*} P=0.027$ Fisher's exact test. (e,f) Tumour growth from all mice in experiment described in $\mathbf{c}{ }^{*} P=0.015$ ANOVA. (g). VX-970 dose-response curves from TOV21G (ARIDIA mutant) and RMG1 (ARID1A wild-type) tumour cells. Cells were exposed to VX-970 for 5 days. ANOVA $P$ value of $<0.0001$. (h) Western blot illustrating PARP-1 cleavage in TOV21G cells exposed to increasing concentrations of VX-970 for $24 \mathrm{~h}$ before cell lysis. As a positive control cells were exposed to camptothecin (Cpt; $1 \mu \mathrm{M}, 24 \mathrm{~h}$ ) before cell lysis. PARP-1, cleaved (Clvd) PARP-1 (85 kDa fragment) and tubulin were detected by western blot. (i) Bar chart illustrating apoptotic fraction in RMG1 and TOV21G cells exposed to increasing concentrations of VX-970 for $24 \mathrm{~h}$. ${ }^{*}$ Student's $\mathrm{t}$-test, $P<0.05$. (j) Schematic representation of VX-970 therapy experiment in mice bearing established TOV21G. Experiment performed as per (a) but with only 28 days treatment. (k) Relative tumour volume plot from (j) showing efficacy of VX-970 in TOV21G xenografts ${ }^{*} P=0.014$, ANOVA. (I). Images of vehicle and VX-970-treated TOV21G xenografts after 28 days treatment. 
type between acute loss of ARID1A expression (via siRNA) as opposed to long-term ARID1A deficiency as is the case in the ARID $1 A^{-/-}$cells. We also noted increased levels of cytoplasmic cyclin B1 in ARID1 $A^{-/-}$cells (Supplementary Fig. $4 \mathrm{H}$ ) a phenotype associated with initiation of a $\mathrm{G}_{2} / \mathrm{M}$ cell cycle checkpoint $^{36}$. Collectively, this data suggested that loss of ARID1A caused a reduced rate of $S$ phase progression and an increased utilization of a $\mathrm{G}_{2} / \mathrm{M}$ cell cycle checkpoint.

Mouse ES cells with an inducible BAF complex defect caused by loss of Brg1 (Smarca4), have delayed progression through a $\mathrm{G}_{2} / \mathrm{M}$ cell cycle checkpoint, a defect in the ability to separate DNA sister chromatids after DNA replication (DNA a
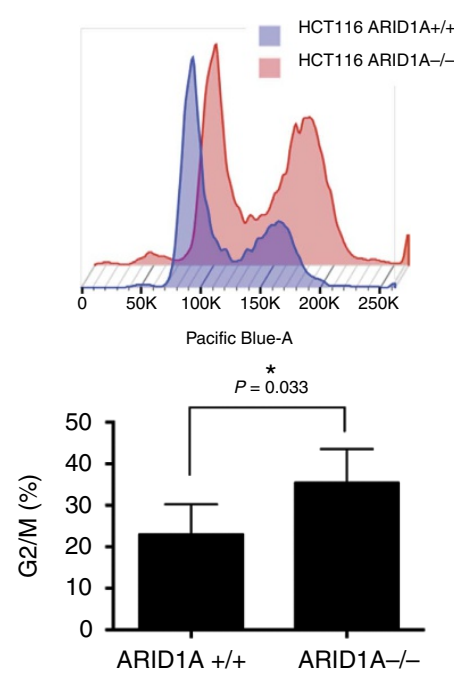

d

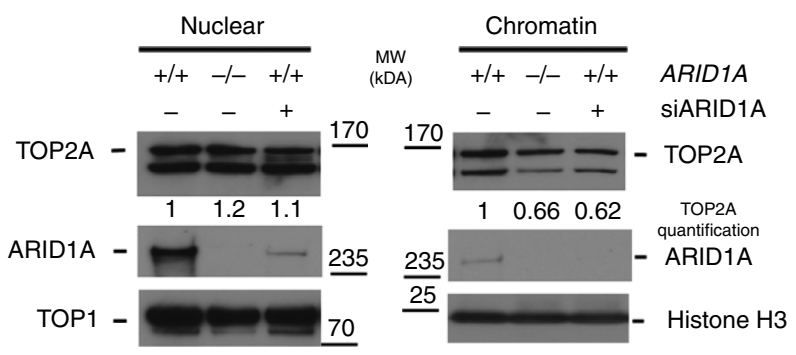

$\mathbf{f}$

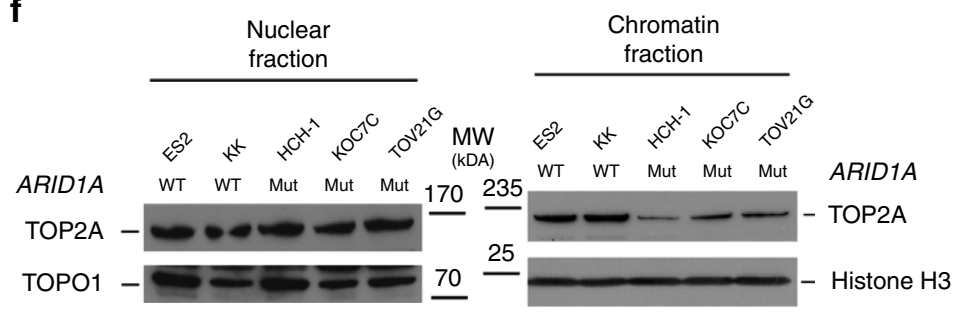

b

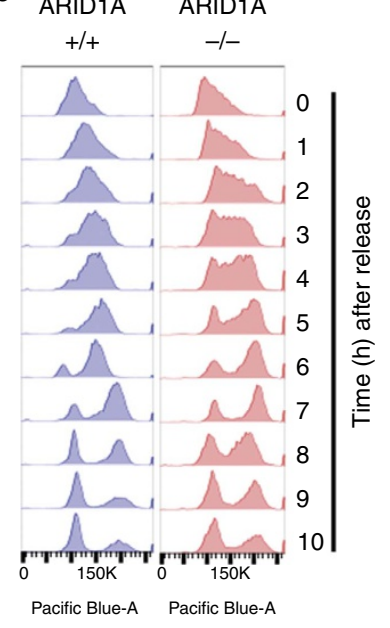

C

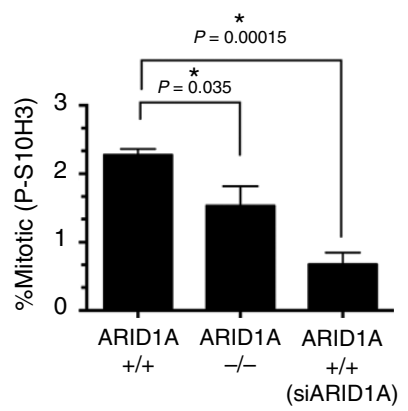

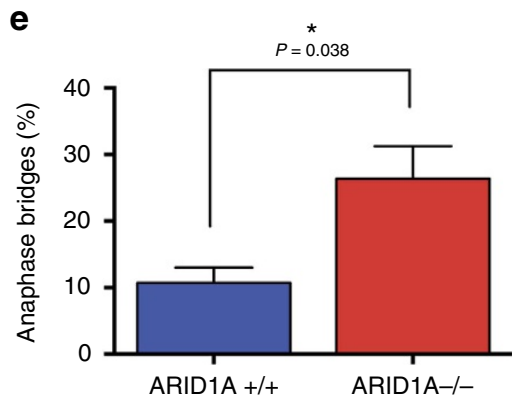

9

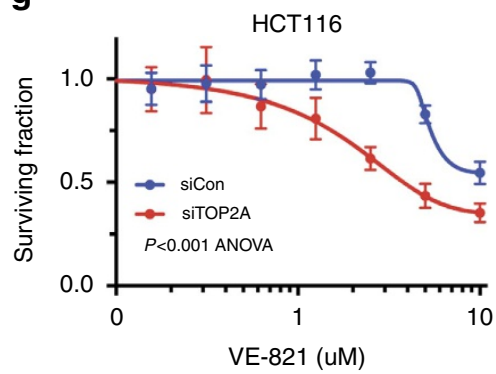

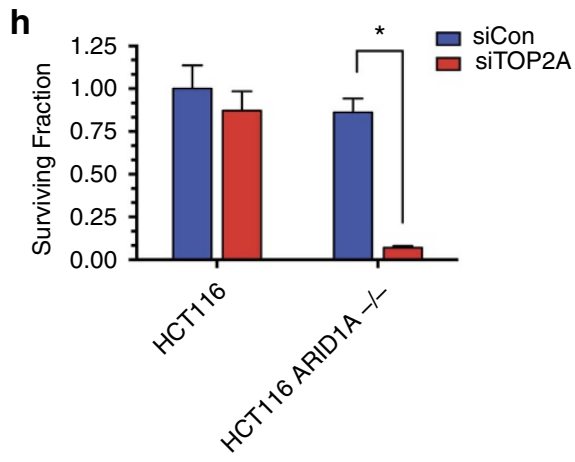


decatenation), and reduced localization of the topoisomerase enzyme, TOP2A, to $\mathrm{DNA}^{37}$. TOP2A modulates the topological structure of DNA and is critical to processes such as chromosome condensation, chromatid separation, DNA transcription and replication (reviewed in refs 38,39). We found that HCT116 $A R I D 1 A^{-1-}$ cells exhibited lower levels of chromatin-bound TOP2A, compared with isogenic ARID1A ${ }^{+/+}$cells (Fig. 4d). In addition RNAi-mediated silencing of ARID1A in ARID1A ${ }^{+/+}$ cells also reduced chromatin-bound TOP2A (Fig. 4d). We also found that the TOP2A defect in ARID1A defective HCT116 cells was associated with an increased frequency of anaphase bridges $(P=0.038$, Student's $t$-test, Fig. $4 \mathrm{e})$, a biomarker of defective DNA decatenation, a process controlled by TOP $2 \mathrm{~A}^{37,40}$. The TOP2A defect was also apparent amongst a panel of OCCC cell lines, where those with ARID1A mutations exhibited a reduction in chromatin-bound TOP2A, despite expressing similar amounts of total TOP2A (Fig. 4f). We also found that siRNA silencing of TOP2A using SMARTPool siRNAs in ARID1A ${ }^{+/+}$HCT116 cells caused sensitivity to both VE-821 and VX-970 $(P<0.001$, ANOVA, Fig. $4 \mathrm{~g}$ and Supplementary Fig. 4I,J). Silencing of TOP2A by SMARTPool siRNA in HCT116 ARID1A ${ }^{-/-}$cells led to high levels of cell death, presumably because of the pre-existing TOP2A defect in these cells (Fig. 4h).

It seemed possible that the defects in TOP2A localization and cell cycle progression in ARID1A mutant cells would generate an enhanced necessity for ATR function that could be therapeutically exploited with ATRi. TOP2A alleviates many of the topological problems caused by chromosomal metabolism such as catenated DNA and DNA transcription/replication interference $^{41}$. It seemed possible that cell division in the presence of a TOP2A defect and a failure to adequately resolve such topological problems could cause genomic instability and ultimately impair the fitness of cells. In HCT116 cell cultures synchronized at the late $G_{1}$ /early $S$ phase boundary using a double thymidine block, we found that exposure to VX-970 accelerated the rate of $\mathrm{G}_{2}$ exit, so that ARID1A $A^{-/-}$cells no longer exhibited the $\mathrm{G}_{2} / \mathrm{M}$ cell cycle progression delay seen in the absence of ATRi (Fig. 5a). In addition, VX-970 exposure also resulted in reduced cytoplasmic Cyclin B1 in both ARID1A ${ }^{+/+}$and $A R I D 1 A^{-/-}$cells (Supplementary Fig. $4 \mathrm{H}$ ). Despite both wild type and ARID1A null cells displaying increased mitotic entry in response to VX970, ATRi exposure led to increased anaphase bridges in ARID1A ${ }^{-/}$cells compared with ARID $1 A^{+/+}(P=0.023$, Student's $t$-test, Fig. $5 \mathrm{~b}$ ) an effect also observed in ARID1A mutant TOV21G cells ( $P=0.006$ by Student's $t$-test, Fig. $5 c$ ). VX-970 exposure also led to a significant enhancement in the frequency of chromosomal aberrations in $A R I D 1 A^{-/-}$cells compared with wild-type cells $(P=0.0007$, Student's $t$-test, Fig. 5d,e). In addition, we found that the VX-970-induced $\gamma \mathrm{H} 2 \mathrm{AX}$ response, a marker of DNA damage, was more pronounced in ARID1A $A^{-/-}$cells (Fig. 5f). A similar $\gamma \mathrm{H} 2 \mathrm{AX}$ response was also observed in ARID1A mutant TOV21G cells (Supplementary Fig. 4G). In ARID1 $A^{-/-}$cells exposed to ATRi for a short period $(2 \mathrm{~h})$, we found an increase in H2AX phosphorylation in Cyclin A positive as well as negative cells (Supplementary Fig. 5A-D). The presence of VX-970-induced $\mathrm{H} 2 \mathrm{AX}$ phosphorylation in $\mathrm{S}$ phase cells was confirmed by western blotting in synchronized cells as they transitioned through $\mathrm{S}$ phase (Supplementary Fig. 5E). Taken together, this suggested that the $\gamma \mathrm{H} 2 \mathrm{AX}$ response to ATRi in ARID1A ${ }^{-/-}$cells occurred in $S / G_{2}$ phases (Cyclin A positive) as well as in $G_{1}$ phase (Cyclin A negative) of the cell cycle, implying that processes occurring in multiple phases of the cell cycle could contribute to the synthetic lethal phenotype observed. It is possible that the $\gamma \mathrm{H} 2 \mathrm{AX}$ response in Cyclin A negative cells could be initiated in $\mathrm{G}_{1}$ itself or could be the result of DNA damage occurring during mitosis becoming apparent as cells transit into $G_{1}$ during the 2-h ATRi exposure. We also noted that VX-970 exposure caused an apoptotic response in ARID1A-deficient cells, as shown by higher caspase-3/7 activation in $A R I D 1 A^{-/-}$cells compared with ARID $1 A^{+/+}$cells $(P<0.05$, Student's $t$-test, Fig. $5 \mathrm{~g})$ and in TOV21G compared with RMG1 cells (Fig. 3i). We also assessed the induction of apoptosis in ATRi exposed cells by measuring PARP-1 cleavage. Camptothecin elicited similar levels of cleavage in both $A R I D 1 A^{-/-}$and $A R I D 1 A^{+/+}$cells, suggesting both genotypes possessed a functional apoptotic response. In contrast, VX-970 caused far higher levels of PARP-1 cleavage in ARID1A ${ }^{-/-}$cells than in ARID1A ${ }^{+/+}$cells (Fig. 5h). From this data we concluded that loss of ARID1A function results in: (i) a defect in the ability of cells to recruit TOP2A to chromatin; and (ii) cell cycle progression defects in both $\mathrm{S}$ and $\mathrm{G}_{2} / \mathrm{M}$ phases of the cell cycle. It seems possible that these factors combined or in isolation might render tumour cells sensitive to small molecule ATRi as these agents impair the ability of cells to mount adequate DDRs, while at the same time accelerating mitotic entry (Fig. 5i).

In addition to the model proposed (Fig. 5i), we also assessed whether other mechanisms of ATRi sensitivity might explain, or at least contribute, to the ARID1A synthetic lethality. The BAF

\footnotetext{
Figure 4 | ARID1A-deficient cells depend on a $\mathbf{G}_{2} / \mathbf{M}$ checkpoint due to a chromosomal decatenation defect. (a) Histogram of the cellular DAPI-stained DNA content, determined by FACS, in asynchronous HCT116 ARID1A $+/+$ (blue) and ARID1A $-/-$ cells (red). Bar chart illustrating the percentage of cells in $\mathrm{G}_{2} / \mathrm{M}$ phase of asynchronously growing $\mathrm{HCT116} A R I D 1 A^{+/+}$and $A R I D 1 A^{-/-}$cells. Asterisk indicates statistical significance $(P=0.033$, Student's $t$-test), error bars represent s.d., from four independent experiments. (b) Histogram of the cellular DAPI-stained DNA content, determined by FACS, in $\mathrm{HCT116} \mathrm{ARID1A}+/+$ and ARID1A ${ }^{-/-}$cells at the indicated time points following release from double thymidine synchronization in $\mathrm{G}_{1} /$ early $\mathrm{S}$ phase. (c) Bar chart illustrating the percentage of mitotic cells with phosphorylation of Histone $\mathrm{H} 3$ on Serine 10. Where shown, HCT116 ARID1A $+/+$ cells were transfected with siARID1A. $48 \mathrm{~h}$ later cells were fixed and stained with FITC-P-S10 Histone H3 and PI. Mitotic cells (P-S10 positive and $2 \mathrm{~N}$ ) were quantified by FACS. Asterisk indicates a statistically significant difference by Student's $t$-test between the indicated comparisons. (d) Western blot of nuclear and chromatin-bound TOP2A. The indicated cell lines were transfected with siRNA targeting ARID1A or control siRNA. Forty-eight hous later, subcellular fractions were isolated and resultant western blots immunoblotted for the indicated proteins. Total amount of TOP2A was quantified and normalized to ARID1A $+/+$ control siRNA, in each fraction. (e) Bar chart illustrating elevated level of anaphase bridges in HCT116 ARID1A ${ }^{-/-}$cells compared with $A R I D 1 A^{+/+}$. A minimum of 50 anaphases were scored in three biological replicate experiments. ${ }^{\star} P=0.038$, Student's $t$-test. (f) Western blot of nuclear and chromatin-bound TOP2A from OCCC cell lines, with the indicated ARID1A status. Fractionation was performed as per (d). (g) Three hundred eighty four-well plate cell survival data from HCT116 cells transfected with siRNA targeting TOP2A (red) or siCon (blue). Twenty four hours after transfection, cells were exposed to VE-821 for 5 continuous days. Error bars represent s.d. Survival curves siTOP2A versus siCon $P$ value $<0.001$, ANOVA. (h). Three hundred eighty four-well plate cell survival data from HCT116 ARID1A $+/+$ and HCT116 ARID1A-/- cells transfected with siRNA targeting TOP2A (red) or siCon (blue). Viability was estimated 5 days after transfection. Error bars represent s.d. and asterisks indicate a statistically significant difference $(P<0.001)$ by Student's $t$-test between the indicated comparisons. FACS, fluorescence-activated cell sorting.
} 
chromatin-remodelling complex plays a central role in the control of gene transcription ${ }^{20,42}$. We therefore also assessed whether loss of ARID1A caused reduced transcription of any previously established ATR synthetic lethal genes that could also explain the ATR/ARID1A synthetic lethal effect. By comparing trans- criptomic profiles of HCT116 ARID1A ${ }^{+/+}$and ARID1A ${ }^{-/-}$ cells (see Methods) we detected a statistically significant reduction in ARID1A mRNA expression in ARID1A ${ }^{-/-}$cells, presumably due to nonsense mediated decay of the mutant transcript (Supplementary Data 4). We did not however, find significant

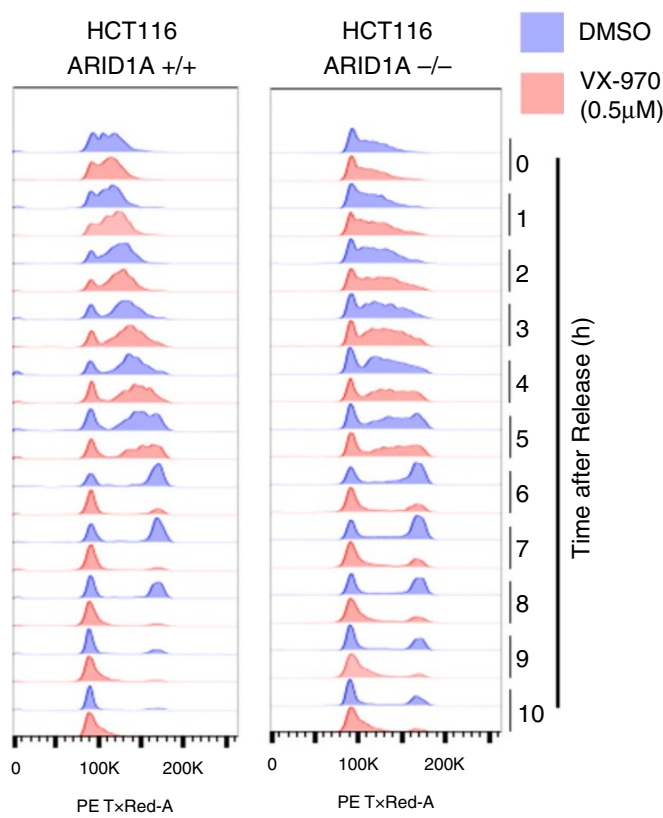

d

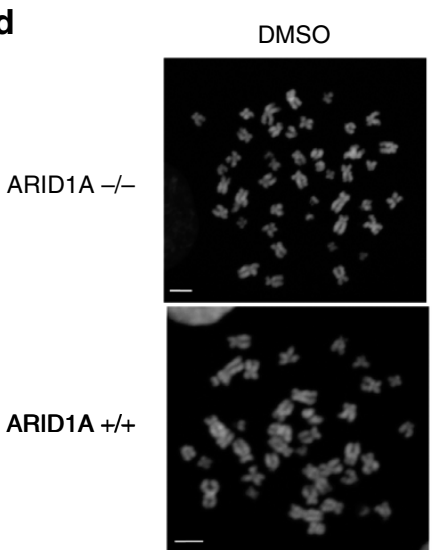

\section{b}

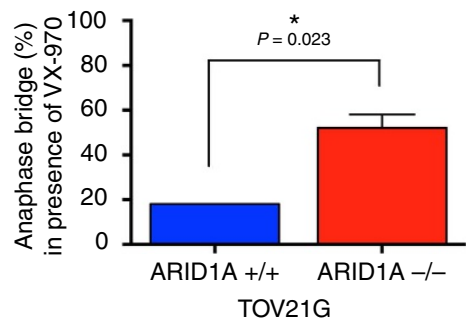

C

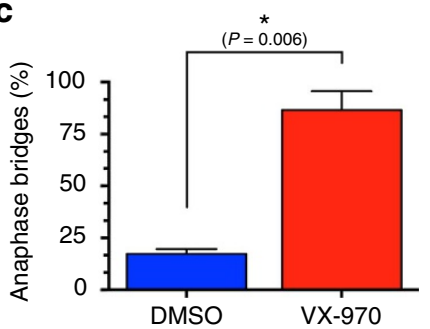

e

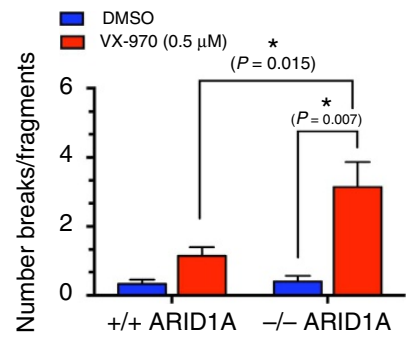

g

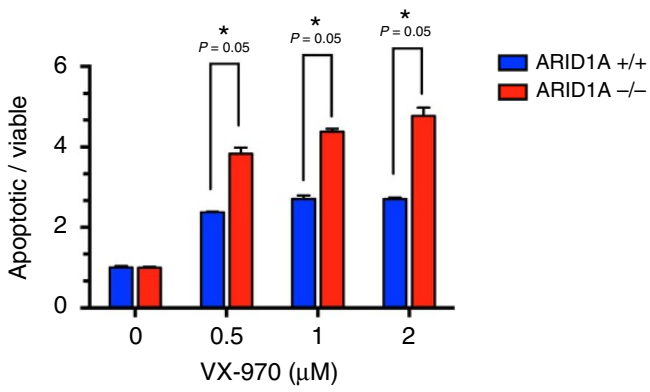

i

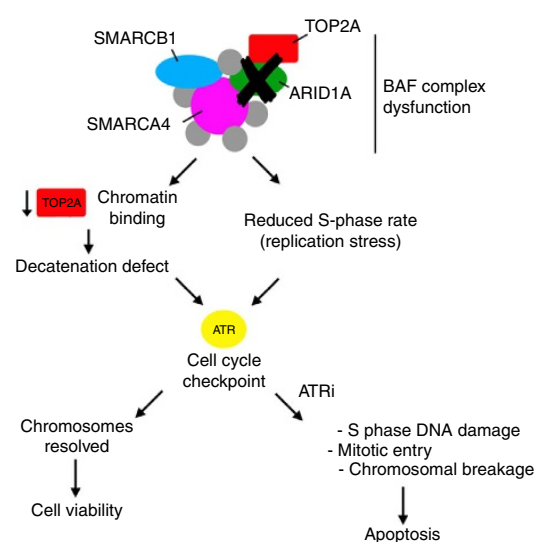


reduction in mRNA levels of $A T M, X R C C 1, A T R$ or the ATR activating genes RAD17, HUS1 or RAD9A in the ARID1A ${ }^{-/-}$ cells when compared with the HCT116 ARID1A ${ }^{+/+}$isogenic model (Supplementary Data 4). ERCC1 defects have previously been associated with ATRi sensitivity ${ }^{12}$ but in this analysis we found ERCC1 transcript levels to be elevated in the ARIDIA defective cells, not decreased (Supplementary Data 4). Although we cannot formally exclude the possibility that a transcriptional mechanism contributes to the ARID1A/ATR synthetic lethality, we were unable to identify significant changes in gene expression in isogenic models that might explain this.

We also assessed whether defects in ATR activity in ARID1A defective cells might explain the synthetic lethal effects observed. Shen et al. recently demonstrated that ATR and ARID1A directly interact and loss of this interaction in HCT116 ARID1A ${ }^{-/-}$ tumour cells results in impaired ATR activation (as measured by ATR autophoshorylation) in response to ionizing radiation, although ATR signalling in response to agents that stall replication forks such as hydroxyurea (HU) and ultraviolet radiation appeared normal ${ }^{32}$. We assessed whether ATR activity was impaired in HCT116 ARID1A $A^{-/-}$tumour cells as well as in a panel of human tumour cell lines characterized according to their ARID1A status (Supplementary Fig. 1A,B). We found that in response to cisplatin, a platinum salt drug that causes replication fork stress ${ }^{43}$, HCT116 ARID1A ${ }^{-/-}$and tumour cell lines with endogenous ARID1A mutations exhibited similar protein expression of ATR to ARID1A wild-type cells and clear ATR p.T1989 autophosphorylation (Supplementary Fig. 1A,B). This data suggested that while ATR responses in HCT116 ARID1A ${ }^{-/-}$tumour cells to IR are impaired ${ }^{32}$, this was not the case in a variety of tumour cells exposed to cisplatin (Supplementary Fig. 1A,B) or other replication fork stalling lesions ${ }^{32}$. As such, we were unable to explain the ATRi sensitivity of ARID1A defective cells by reason of reduced ATR activity.

\section{Discussion}

Our data suggest that ATRi could have potential as single-agent treatments for ARID1A defective cancers. The highly recurrent nature of ARID1A mutations in human cancer and the availability of clinical ATRi suggests that once Phase I clinical trials are complete, biomarker driven proof-of-concept trials could be instigated to assess this hypothesis. These trials could be conducted in cancer types where there is a high frequency of ARID1A mutations, such as OCCC, where standard of care therapeutic responses are limited and where few targeted approaches exist.
In Fig. 5i, we present a working model to explain the ARID1A/ ATR synthetic lethality. There might of course be additional mechanisms that also contribute to this synthetic lethality, as highlighted earlier. Although we cannot find evidence for ATR activation defects in ARIDIA defective cells, we cannot formally exclude the possibility that these processes play a part in the synthetic lethal effect and act in parallel to the TOP2A and cell cycle defects. In addition, cells utilizing the ALT-pathway of telomere maintenance have also been shown to have increased ATRi sensitivity ${ }^{44}$, although whether this effect operates in all tumour cells with an ALT defect is not clear ${ }^{45}$. To our knowledge, none of the models used here are ALT-positive. In addition, in Fig. 4, we show that ARID1A defective cells have a delayed progression through the cell cycle and specifically delayed progression through $\mathrm{S}$ and $\mathrm{G}_{2} / \mathrm{M}$. Cell cycle defects appear to be an inherent characteristic of ARID1A/BAF defective cells, as originally noted by others ${ }^{31,37}$; as such, it seems difficult to eliminate the possibility that some other characteristic(s) associated with reduced proliferation might also influence the sensitivity of ARID1A mutant cells to ATRi. Topoisomerase function has also been implicated in the prevention of deleterious collisions between the transcriptional and replication machineries ${ }^{41}$, potentially providing another mechanism that, if defective, could lead to a dependency upon ATR, and could explain the $\mathrm{S}$ phase delay in ARID1A $\mathrm{A}^{-/}$- cells. Finally it is possible that loss of ARID1A, by causing alterations in chromatin structure that lead to an altered transcriptional programme, could impair the expression of other genes critical for cells to survive in the face of ATR inhibition. In this study we compared the transcriptional profiles of HCT116 ARID1A A $^{-/-}$and ARID1A ${ }^{+1+}$ cells but observed no clear defects in the transcript expression of genes known to be associated with ATRi sensitivity (Supplementary Data 4).

Our results, and previous work ${ }^{37}$ have detailed a role for ARID1A in chromosomal decatenation through the appropriate localization of TOP2A to chromatin. However, despite this defect in TOP2A, tumour subtypes characterized by a high frequency of ARID1A mutations, such as OCCC, have been reported to have relatively 'flat' genomes (that is, relatively few large-scale genomic alterations/rearrangements ${ }^{46}$ compared with other gynaecological malignancies such as high-grade serous ovarian cancers. While the TOP2A defect and 'flat' genomes might possibility be viewed as inconsistent with one another, it is possible that the TOP2A defect in ARID1A mutant tumours has relatively moderate effects (compared with a profound homologous recombination defect for example) on the structure of the genome as it is normally kept in check by proteins such as ATR. Indeed, when

\footnotetext{
Figure 5 | ATRi effect on cell cycle progression, chromosomal instability, DNA damage and apoptosis. (a). Histogram of the cellular DAPI-stained DNA content, determined by FACS, in HCT116 ARID1A ${ }^{+/+}$and $A R I D 1 A^{-/-}$cells at the indicated time points following release from synchronization in $\mathrm{G}_{1} /$ early $\mathrm{S}$ phase by double thymidine block. Cells were released from thymidine block into media containing DMSO (blue) or VX-970 (0.5 $\mu \mathrm{M}$, red). (b). Bar chart illustrating increased level of anaphase bridges in HCT116 ARID1A ${ }^{-/-}$and ARID1A ${ }^{+/+}$cells exposed to VX-970 $(0.5 \mu \mathrm{M}, 8 \mathrm{~h})$. Cells were stained with DAPI. A minimum of 50 anaphases were scored in three biological replicate experiments. ${ }^{\star} P=0.023$, Student's $t$-test. (c). Bar chart illustrating frequency of anaphase bridges in TOV21G cells exposed to VX-970 $(0.5 \mu \mathrm{M})$ or DMSO for $8 \mathrm{~h}$ before fixation. Experiment performed as for $(\mathbf{b})$. $P=0.006$ Student's t-test. (d) Images of mitotic spreads from HCT116 ARID1A ${ }^{+/+}$and ARID1A-/- cells following exposure to either DMSO or VX-970 (1 $\left.\mu \mathrm{M}\right)$. Scale bar, $20 \mu \mathrm{m}$. (e) Bar chart illustrating extent of chromosomal abnormalities in HCT116 ARID1A ${ }^{+/+}$and ARID1A ${ }^{-/-}$cells exposed to VX-970 $(1 \mu \mathrm{M})$. ${ }^{\star} P<0.05$, Student's t-test. (f) Western blot illustrating $\gamma \mathrm{H} 2 \mathrm{AX}$ in HCT116 ARID1A $+/+$ and ARID1A ${ }^{-/-}$cells exposed to VX-970 (0.5 $\left.\mu \mathrm{M}\right)$ for the indicated time before cell lyses. (g) Bar chart illustrating apoptotic fraction in cells exposed to increasing concentrations of VX-970 for $24 \mathrm{~h}$. Experiment as per Fig. $3 \mathrm{i}$. (h) Western blot illustrating PARP cleavage in HCT116 ARID1A $+/+$ and ARID1A ${ }^{-/}$cells exposed to VX-970. Experiment performed as per Fig. 3h. (i) A model for the proposed mechanism driving the sensitivity of ARID1A-deficient cells to ATRi. Loss of ARID1A function results in: (i) a defect in the ability of cells to recruit TOP2A to chromatin; and (ii) cell cycle progression defects in both $S$ and $G_{2} / M$ phases of the cell cycle. These factors combined or in isolation might render tumour cells sensitive to small molecule ATRi as these agents impair the ability of cells to mount adequate DDRs, while at the same time accelerating mitotic entry. FACS, fluorescence-activated cell sorting.
} 
ARID1A defective cells are exposed to an ATRi, chromosomal rearrangements are observed (Fig. 5d,e). We do note that the potential for ATR inhibition to alter the genomic stability of ARID1A mutant tumours in a genotype specific manner (and therefore potentially tumour cell specific manner) might have beneficial therapeutic effects but could also have deleterious effects in driving tumourigenesis or drug resistance by the generation of new oncogenic mutations and chromosomal rearrangements. This is a challenge not just to the use of ATRi but is also an issue of concern when using other agents whose mechanism of action results in changes to the structure of the genome. This issue could be addressed by assessing what additional processes are required to ensure that any increase in genomic instability results in tumour cell death, rather than cell survival with a reordered genome.

Finally, we also note that the precise composition of the BAF complex and the influence of other driver mutations in ARID1A mutant tumours might also be important in determining the ARID1A/ATR synthetic lethality and our subsequent work will focus on assessing how the balance between the different members of the SWI/SNF complex might modulate the TOP2A defect and ATRi sensitivity.

\section{Methods}

Cell lines. ES2 and TOV21G were obtained from the American Type Tissue Collection. RMG-1, SMOV2, KOC7C, HCH1, OVAS, OVISE, OVMANA, OVTOKO, OVSAYO and KK were courtesy of Dr Hiroaki Itamochi (Tottori University School of Medicine, Yonago, Japan). OCCC lines were grown in McCoys with $10 \%$ fetal calf serum (FCS). The identity of cell lines was confirmed by short tandem repeat typing using the StemElite Kit (Promega) in March 2013 and yearly thereafter. ARID1A HCT116 isogenic cell lines were obtained from Horizon Discovery and grown in McCoys with 10\% FCS. Aridla null and wild-type mouse ES cells were obtained from Dr Zhong Wang (Harvard Medical School, USA) and grown on gelatin coated plates in DMEM with $10 \%$ FCS supplemented with $0.1 \mathrm{mM}$ NEAA, $1 \mathrm{mM}$ sodium pyruvate, $0.1 \mathrm{mM} \beta$-mercaptothanol and $2,000 \mathrm{U} \mathrm{LIF} \mathrm{ml}^{-1}$

VE-821 siRNA screen. A siRNA library (1,280 siRNAs listed in Supplementary Data 1) was purchased from Dharmacon. Genes were selected as described in the main text. Each well contained a SMART pool of four distinct siRNA species targeting different sequences of the target transcript. Each plate was supplemented with negative siCONTROL (12 wells; Dharmacon) and positive control (four wells, siPLK1, Dharmacon). RNAi screening conditions were optimized and raw CellTitre-Glo (Promega) luminescent viability readings were generated as previously described ${ }^{30}$. VE-821 or vehicle (DMSO) was added $24 \mathrm{~h}$ after transfection at $1 \mu \mathrm{M}$ concentration in media and cells were exposed for 5 days. Statistical analysis of the siRNA screen was performed as described elsewhere ${ }^{30}$. In brief, luminescence values from CellTitre-Glo assays in ATRi and DMSO exposed cells were $\log _{2}$ transformed and then normalized to plate median (PM) effects. Drug Effect (DE) scores were calculated from PM normalized data using the equation: $\mathrm{DE}=\left(\log _{2}\right.$ PM normalized signal of siRNA in the presence of ATRi $)-$ $\left(\log _{2}\right.$ PM normalized signal of siRNA in the absence of ATRi). DE values were then $Z$-score standardized according to screen median and median absolute deviation values.

Chemicals. The ATRi VE- 821 and VX-970 were provided by Vertex Pharmaceuticals. Olaparib and BMN673 were purchased from Selleck Chemicals; cisplatin was purchased from Sigma.

Western blotting and antibodies. Whole-cell protein extracts were prepared from cells lysed in NET-N buffer (20 mM Tris pH 7.6, 1 mM EDTA, 1\% NP40, $150 \mathrm{mM} \mathrm{NaCl}$ ) supplemented with protease inhibitor cocktail tablets (Roche, West Sussex, UK). Western blots were carried out with precast Bis-Tris gels (Invitrogen, Paisley, UK). The following primary antibodies were used in this study; ARID1A (1:1,000, Cell Signaling, D2A8U), ATR (1:500, Santa Cruz, sc1887), P-T1989 ATR (1:1,000, Gene Tex, GTX128145), Tubulin (1:10,000, Sigma, T6074), $\gamma \mathrm{H} 2 \mathrm{AX}$ (1:3,000, Cell Signaling, 2,577), PARP-1 (1:3,000, Santa Cruz, sc-8007), Ezrin (1:3,000, Cell Signaling, 3,145), TOP2A (1:1,000, Cell Signaling, D10G9), Cyclin B1 (1:3,000, Cell Signaling, 4,138), Histone H3 (1:3,000, Cell Signaling, $9,715)$ and Lamin A/C (1:3,000, Cell Signaling, 4,777). Uncropped scans for all western blots shown in Figs 1-5 are shown in Supplementary Figs 6-10.
Cellular viability assays. Short-term survival assays were performed in 384-well plates. Exponentially growing cells were plated at a concentration of 500 cells per well. Drug was added $24 \mathrm{~h}$ after seeding and cells were continuously exposed to the drug for 5 days, after which cell viability was estimated using CellTitre-Glo luminescence (Promega). For clonogenic assays, cells were seeded in six-well plates (500 cells per well) and continuously exposed to drug $24 \mathrm{~h}$ after seeding for 14 days. Media containing fresh drug was replaced every $72 \mathrm{~h}$. Cells were fixed with $10 \%$ trichloroacetic acid and stained with sulphorhodamine B (Sigma-Aldrich, Gillingham, UK). Colonies were counted manually. All cell-based assays were performed at least in triplicate. Lines of best fit were plotted using a four-parameter nonlinear regression or linear models where appropriate.

Cell cycle analysis. Cells were plated at a density of $2 \times 10^{5}$ cells per well of a six-well plate and incubated for $24 \mathrm{~h}$ after which ATRi or $0.1 \%$ DMSO was added for the indicated period of time. After incubation, adherent cells were harvested and then fixed with cold $50(\mathrm{v} / \mathrm{v}) \%$ ethanol in PBS. Cells were then treated with RNase A for $30 \mathrm{~min}$ before nucleic acid staining with propidium iodide (PI, Sigma). Samples were analysed on a BD LSR II flow cytometer using BD FACSDiva software (BD Biosciences). For synchronization experiments cells were incubated with $2 \mathrm{mM}$ thymidine for $12 \mathrm{~h}$, washed and incubated with media for $16 \mathrm{~h}$, then incubated in $2 \mathrm{mM}$ thymidine for a further $12 \mathrm{~h}$. Cells were then released into media containing either VX-970 or DMSO and samples fixed as above for cell cycle analysis.

p-H3 assay. Following experimental treatment, cells were fixed using cold $50 \%$ ethanol and PBS. Before analysis, cells were centrifuged and resuspended in $1 \mathrm{ml}$ $0.25 \%(\mathrm{v} / \mathrm{v})$ solution of Triton-X100 in PBS for $15 \mathrm{~min}$. Following centrifugation, cells were resuspended in $100 \mu \mathrm{l}$ of PBS solution containing $1 \%(\mathrm{w} / \mathrm{v})$ bovine serum albumin (BSA) and $0.75 \mu \mathrm{g}$ of P-S10 Histone H3 antibody (Jackson ImmunoResearch). Samples were incubated at room temperature for $3 \mathrm{~h}$. Samples were then centrifuged and washed with PBS solution containing $1 \%$ BSA. Cells were subsequently suspended in $100 \mu \mathrm{l}$ of FITC-labelled goat anti-rabbit secondary antibody (Jackson ImmunoResearch) in a 1:30 dilution in PBS with 1\% BSA. Samples were incubated in the dark for $30 \mathrm{~min}$ and resuspended in PBS solution containing PI $\left(5 \mu \mathrm{g} \mathrm{ml}^{-1}\right)$ and RNase $\left(1 \mathrm{mg} \mathrm{ml}^{-1}\right)$. Samples were analysed on a BD LSR II flow cytometer using BD FACSDiva software (BD Biosciences).

Apoptosis assay. HCT116 isogenic and OCCC cells were plated in a 96-well plate at high density (15-20,000 per well). Twenty four hours later serial dilutions of VX-970 were added for the indicated period of time. ApoTox-Glo Triplex Assay (Promega) was performed as per the manufactures protocol.

In vivo efficacy studies. In vivo efficacy studies were performed using HCT116 ARID1A ${ }^{+/+}$, HCT116 ARID1A ${ }^{-/-}$and TOV21G cells injected subcutaneously in the flank of female CD-1 Nude mice. Animals were treated with either vehicle alone (10\% D- $\alpha$-Tocopherol polyethylene glycol 1000 succinate) or VX-970 ( $60 \mathrm{mg} \mathrm{kg}^{-1}$ in $10 \% \mathrm{D}-\alpha$-Tocopherol polyethylene glycol 1000 succinate) by oral gavage. Treatment was administered four times weekly for the indicated length of time. Tumours were measured manually by calliper trice weekly and animals were sacrificed when tumours reached $>15 \mathrm{~mm}$ in any direction. All in vivo modelling was carried out according to ARRIVE guidelines, regulations set out in the UK Animals (Scientific Procedures) Act 1986, and in line with a UK Home Office approved project licence held by CJL and approved by the ICR ethics board.

Anaphase bridge analysis. Cells were grown on Poly-lysine-coated coverslips for $24 \mathrm{~h}$ before exposure to the indicated treatment. Samples were fixed in formaldehyde (4\%), permeablized in Triton X-100 $(0.2 \%)$ and DNA stained with DAPI. Slides were then imaged at $60 \mathrm{X}$ on a Leica confocal microscope.

Mitotic spreads. Following exposure to the indicated treatment, cells were incubated with $0.5 \%$ colchicine for $4 \mathrm{~h}$. Cells were harvested, washed in PBS and incubated in $0.56 \% \mathrm{KCl}$ at $37 \mathrm{C}$ for $20 \mathrm{~min}$. Samples were then fixed (3 methanol:1 acetic acid) and DAPI was added. Cell solutions were dropped onto clean coverslips and mitotic spreads imaged at $\times 60$ on a Leica confocal microscope.

Transcriptomic analaysis. Whole transcriptome analysis was performed on a Illumina BeadArray HumanHT-12 v4 device at the Wellcome Trust Centre for Human Genetics (University of Oxford). Three biological replicates of HCT116 ARIDIA $^{+/+}$and ARID1A ${ }^{-/-}$were analysed. Differential expression and statistical analysis was performed using the LIMMA package from the Bioconductor project ${ }^{47}$

Immunofluorescence analysis of $\gamma \mathbf{H 2 A X}$. Cells were grown on poly-lysinecoated coverslips for $24 \mathrm{~h}$ before treatment. Following treatment cells were fixed in $4 \%$ paraformaldehyde and permeablized with $0.2 \%$ Triton X-100. Cells were stained with antibodies to $\gamma \mathrm{H} 2 \mathrm{AX}$ (Millipore, 05-636), Cyclin A (Abcam, ab181591) and DAPI. Fluorescently labelled secondary antibodies were 
incubated $30 \mathrm{~min}$ before mounting cells on slides. Slides were imaged at $\times 40$ on a Leica confocal microscope.

Data availability. All relevant data not presenting in the main figures or Supplementary Data is available from the authors.

\section{References}

1. Shiloh, Y. \& Ziv, Y. The ATM protein kinase: regulating the cellular response to genotoxic stress, and more. Nat. Rev. Mol. Cell Biol. 14, 197-210 (2013).

2. Zou, L. \& Elledge, S. J. Sensing DNA damage through ATRIP recognition of RPA-ssDNA complexes. Science 300, 1542-1548 (2003).

3. Moynahan, M. E. \& Jasin, M. Mitotic homologous recombination maintains genomic stability and suppresses tumorigenesis. Nat. Rev. Mol. Cell Biol. 11, 196-207 (2010)

4. Zeman, M. K. \& Cimprich, K. A. Causes and consequences of replication stress. Nat. Cell Biol. 16, 2-9 (2014).

5. Karnitz, L. M. \& Zou, L. Molecular pathways: targeting ATR in cancer therapy. Clin. Cancer Res. 21, 4780-4785 (2015).

6. Toledo, L. I. et al. A cell-based screen identifies ATR inhibitors with synthetic lethal properties for cancer-associated mutations. Nat. Struct. Mol. Biol. 18, 721-727 (2011)

7. Foote, K. M. et al. Discovery of 4-\{4-[(3R)-3-Methylmorpholin-4-yl]-6-[1(methylsulfonyl)cyclopropyl]pyrimidin-2-y l\}-1H-indole (AZ20): a potent and selective inhibitor of ATR protein kinase with monotherapy in vivo antitumor activity. J. Med. Chem. 56, 2125-2138 (2013).

8. Pires, I. M. et al. Targeting radiation-resistant hypoxic tumour cells through ATR inhibition. Br. J. Cancer 107, 291-299 (2012).

9. Reaper, P. M. et al. Selective killing of ATM- or p53-deficient cancer cells through inhibition of ATR. Nat. Chem. Biol. 7, 428-430 (2011).

10. Josse, R. et al. ATR inhibitors VE-821 and VX-970 sensitize cancer cells to topoisomerase $\mathrm{i}$ inhibitors by disabling DNA replication initiation and fork elongation responses. Cancer Res. 74, 6968-6979 (2014).

11. Huntoon, C. J. et al. ATR inhibition broadly sensitizes ovarian cancer cells to chemotherapy independent of BRCA status. Cancer Res. 73, 3683-3691 (2013).

12. Mohni, K. N., Kavanaugh, G. M. \& Cortez, D. ATR pathway inhibition is synthetically lethal in cancer cells with ERCC1 deficiency. Cancer Res. 74, 2835-2845 (2014).

13. Sultana, R. et al. Ataxia telangiectasia mutated and Rad3 related (ATR) protein kinase inhibition is synthetically lethal in XRCC1 deficient ovarian cancer cells. PLOS ONE 8, e57098 (2013).

14. Ruiz, S. et al. A genome-wide CRISPR screen identifies CDC25A as a determinant of sensitivity to ATR inhibitors. Mol. Cell 62, 307-313 (2016).

15. Kwok, M. et al. Synthetic lethality in chronic lymphocytic leukaemia with DNA damage response defects by targeting the ATR pathway. Lancet 385 (Suppl 1), S58 (2015).

16. Kwok, M. et al. ATR inhibition induces synthetic lethality and overcomes chemoresistance in TP53 or ATM defective chronic lymphocytic leukemia cells. Blood 127, 582-595 (2015).

17. Kadoch, C. et al. Proteomic and bioinformatic analysis of mammalian SWI/ SNF complexes identifies extensive roles in human malignancy. Nat. Genet. 45, 592-601 (2013)

18. Mueller-Planitz, F., Klinker, H. \& Becker, P. B. Nucleosome sliding mechanisms: new twists in a looped history. Nat. Struct. Mol. Biol. 20, 1026-1032 (2013)

19. Jeggo, P. A. \& Downs, J. A. Roles of chromatin remodellers in DNA double strand break repair. Exp. Cell Res. 329, 69-77 (2014).

20. Hohmann, A. F. \& Vakoc, C. R. A rationale to target the SWI/SNF complex for cancer therapy. Trends Genet. 30, 356-363 (2014).

21. Wang, X. et al. Two related ARID family proteins are alternative subunits of human SWI/SNF complexes. Biochem. J. 383, 319-325 (2004).

22. Wilson, B. G. \& Roberts, C. W. SWI/SNF nucleosome remodellers and cancer. Nat. Rev. Cancer 11, 481-492 (2011).

23. Llona-Minguez, S., Hoglund, A., Jacques, S. A., Koolmeister, T. \& Helleday, T. Chemical strategies for development of ATR inhibitors. Expert Rev. Mol. Med. 16, e10 (2014).

24. Nghiem, P., Park, P. K., Kim Ys, Y. S., Desai, B. N. \& Schreiber, S. L. ATR is not required for p53 activation but synergizes with p53 in the replication checkpoint. J. Biol. Chem. 277, 4428-4434 (2002).

25. Sangster-Guity, N., Conrad, B. H., Papadopoulos, N. \& Bunz, F. ATR mediates cisplatin resistance in a p53 genotype-specific manner. Oncogene 30, 2526-2533 (2011).

26. Liu, S. et al. ATR autophosphorylation as a molecular switch for checkpoint activation. Mol. Cell 43, 192-202 (2011).

27. Nam, E. A. et al. Thr-1989 phosphorylation is a marker of active ataxia telangiectasia-mutated and Rad3-related (ATR) kinase. J. Biol. Chem. 286, 28707-28714 (2011)

28. Futreal, P. A. et al. A census of human cancer genes. Nat. Rev. Cancer 4, 177-183 (2004)
29. Wood, R. D., Mitchell, M. \& Lindahl, T. Human DNA repair genes, 2005. Mutat. Res. 577, 275-283 (2005).

30. Lord, C. J., McDonald, S., Swift, S., Turner, N. C. \& Ashworth, A. A highthroughput RNA interference screen for DNA repair determinants of PARP inhibitor sensitivity. DNA Repair (Amst.) 7, 2010-2019 (2008).

31. Gao, X. et al. ES cell pluripotency and germ-layer formation require the SWI/SNF chromatin remodeling component BAF250a. Proc. Natl Acad. Sci. USA 105, 6656-6661 (2008).

32. Shen, J. et al. ARID1A deficiency impairs the DNA damage checkpoint and sensitizes cells to PARP inhibitors. Cancer Discov. 5, 752-767 (2015).

33. Watanabe, R. et al. SWI/SNF factors required for cellular resistance to DNA damage include ARID1A and ARID1B and show interdependent protein stability. Cancer Res. 74, 2465-2475 (2014).

34. Brough, R. et al. Functional viability profiles of breast cancer. Cancer Discov. 1, 260-273 (2011).

35. Campbell, J. et al. Large scale profiling of kinase dependencies in cancer cell lines. Cell Rep. 14, 2490-2501 (2016).

36. Greer Card, D. A., Sierant, M. L. \& Davey, S. Rad9A is required for G2 decatenation checkpoint and to prevent endoreduplication in response to topoisomerase II inhibition. J. Biol. Chem. 285, 15653-15661 (2010).

37. Dykhuizen, E. C. et al. BAF complexes facilitate decatenation of DNA by topoisomerase IIalpha. Nature 497, 624-627 (2013).

38. Champoux, J. J. DNA topoisomerases: structure, function, and mechanism. Annu. Rev. Biochem. 70, 369-413 (2001).

39. Chen, T., Sun, Y., Ji, P., Kopetz, S. \& Zhang, W. Topoisomerase IIalpha in chromosome instability and personalized cancer therapy. Oncogene 34, 4019-4031 (2015)

40. Johnson, M., Phua, H. H., Bennett, S. C., Spence, J. M. \& Farr, C. J. Studying vertebrate topoisomerase 2 function using a conditional knockdown system in DT40 cells. Nucleic Acids Res. 37, e98 (2009).

41. Bermejo, R. et al. Top1- and Top2-mediated topological transitions at replication forks ensure fork progression and stability and prevent DNA damage checkpoint activation. Genes Dev. 21, 1921-1936 (2007).

42. Brownlee, P. M., Meisenberg, C. \& Downs, J. A. The SWI/SNF chromatin remodelling complex: its role in maintaining genome stability and preventing tumourigenesis. DNA Repair (Amst.) 32, 127-133 (2015).

43. Siddik, Z. H. Cisplatin: mode of cytotoxic action and molecular basis of resistance. Oncogene 22, 7265-7279 (2003).

44. Flynn, R. L. et al. Alternative lengthening of telomeres renders cancer cells hypersensitive to ATR inhibitors. Science 347, 273-277 (2015).

45. Deeg, K. I., Chung, I., Bauer, C. \& Rippe, K. Cancer cells with alternative lengthening of telomeres do not display a general hypersensitivity to ATR inhibition. Front. Oncol. 6, 186 (2016).

46. Kuo, K. T. et al. DNA copy numbers profiles in affinity-purified ovarian clear cell carcinoma. Clin. Cancer Res. 16, 1997-2008 (2010).

47. Lavoie, T. N. et al. IL-22 regulation of functional gene expression in salivary gland cells. Genomics Data 7, 178-184 (2016).

\section{Acknowledgements}

We thank Dr Zhong Wang (Harvard Medical School, USA) for providing Arid1a null and wild-type mouse ES cells. This work was funded by Breast Cancer Now as part of their funding to the Breast Cancer Now Toby Robins Breast Cancer Research Centre, Cancer Research UK (grant number C347/A8363) and Vertex Pharmaceuticals. We acknowledge NHS funding to the NIHR Royal Marsden Hospital Biomedical Research Centre. CJR is a Sir Henry Wellcome Fellow jointly funded by Science Foundation Ireland, the Health Research Board, and the Wellcome Trust (grant number 103049/Z $13 / Z)$ under the SFI-HRB-Wellcome Trust Biomedical Research Partnership.

\section{Author contributions}

C.T.W., R.M., H.N.P., S.E.J., R.R. and R.B. designed, conducted and analysed in vitro cellbased experiments. C.T.W., J.F., A.K., N.B., P.B.V. and A.R.R. conducted and analysed in vivo experiments. C.T.W., R.M., J.C., A.G., C.J.R. analysed high-throughput screen data. P.M.R. and J.R.P. developed and provided small molecule inhibitors and advised on their use. A.A. and C.J.L. directed the research and secured funding. All authors contributed to the writing of the manuscript and approved the final version.

\section{Additional information}

Supplementary Information accompanies this paper at http://www.nature.com/ naturecommunications

Competing financial interests: P.M.R. and J.R.P. are paid employees of Vertex Pharmaceuticals. Part of this work has been funded by Vertex Pharmaceuticals as part of a Sponsored Research Agreement between Vertex and The Institute of Cancer Research, London. 
Reprints and permission information is available online at http://npg.nature.com/ reprintsandpermissions/

How to cite this article: Williamson, C. T. et al. ATR inhibitors as a synthetic lethal therapy for tumours deficient in ARID1A. Nat. Commun. 7, 13837 doi: 10.1038/ncomms13837 (2016).

Publisher's note: Springer Nature remains neutral with regard to jurisdictional claims in published maps and institutional affiliations. (c) (i) This work is licensed under a Creative Commons Attribution 4.0 International License. The images or other third party material in this article are included in the article's Creative Commons license, unless indicated otherwise in the credit line; if the material is not included under the Creative Commons license, users will need to obtain permission from the license holder to reproduce the material. To view a copy of this license, visit http://creativecommons.org/licenses/by/4.0/

(C) The Author(s) 2016 